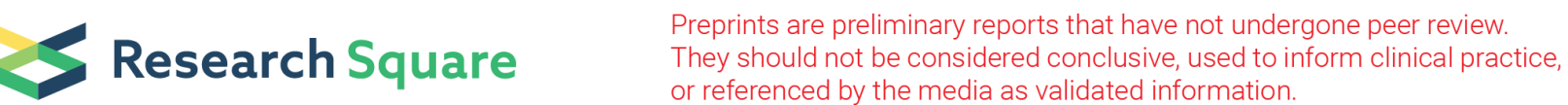

\section{Association between H-type hypertension and Cognitive impairment in Kailuan community of China: A cross-sectional study.}

\section{Xueyu Chen}

Shandong First Medical University \& Shandong Academy of Medical Sciences https://orcid.org/00000001-6104-4240

Hui Xi

Peking University International Hospital

\section{Long Ji}

Shandong First Medical University \& Shandong Academy of Medical Sciences

\section{Weijia Xing}

Shandong First Medical University \& Shandong Academy of Medical Sciences

\section{Haifeng Hou}

Shandong First Medical University \& Shandong Academy of Medical Sciences

\section{Yanru Chen}

Shandong First Medical University \& Shandong Academy of Medical Sciences

\section{Xiaohui Wang}

Shandong First Medical University \& Shandong Academy of Medical Sciences

\section{Zhaoyang Tang}

Shandong First Medical University \& Shandong Academy of Medical Sciences Jian LV

Shandong First Medical University \& Shandong Academy of Medical Sciences

\section{Tan Tan}

Shandong First Medical University \& Shandong Academy of Medical Sciences

\section{Guoyong Ding}

Shandong First Medical University \& Shandong Academy of Medical Sciences

\section{Qingzhi Hou}

Shandong First Medical University \& Shandong Academy of Medical Sciences

Dong Li ( $\nabla$ tsmcdongli@163.com )

\section{Research}

Keywords: Hypertension, $\mathrm{H}$-type hypertension, hyperhomocysteinemia, cognitive impairment, risk factors 
Posted Date: December 19th, 2019

DOI: https://doi.org/10.21203/rs.2.19277/v1

License: (c) (i) This work is licensed under a Creative Commons Attribution 4.0 International License. Read Full License 


\section{Abstract}

\section{Background}

Hypertension (HTN) and hyperhomocysteinemia (HHcy) have been reported to be independent predictors of cognitive impairment. However, the conclusions were inconsistent and few studies had reported the combination prediction of HTN and HHcy for cognitive impairment. Our study aimed to evaluate the association between H-type HTN, HTN and HHcy and the risk of cognitive impairment.

Methods

We used a cross-sectional study- participants aged over 40 years, which were selected from Kailuan communities in China. Cognitive impairment was determined by MMSE scale (MMSE<24). Multivariable logistic regression was used to analyze the association of H-type HTN and cognitive impairment, and we also made stratification analysis by age of participants.

Results

A total of 3454 participants were included in our study, and $\geq 65$ years old was $16.79 \%$; the gender of male was $56.77 \%$. The prevalence of cognitive impairment in the H-type HTN $(\mathrm{HTN}+/ \mathrm{HHcy}+)$ group $(10.74 \%)$ was significantly higher than other three groups $(2.92 \%, 6.42 \%, 5.86 \%$, respectively). The logistic regression results showed that participants with $\mathrm{H}$-type HTN was positively associated with cognitive impairment risk ( $\mathrm{OR}=2.57,95 \% \mathrm{Cl}: 1.38-4.80)$. Similar results were observed in participants stratified by age (65 years) ( $<65$ years old: $\mathrm{OR}=2.01,95 \% \mathrm{Cl}: 0.87-4.67 ; \geq 65$ years old: $\mathrm{OR}=3.63,95 \% \mathrm{Cl}: 1.32-10.01$ ).

\section{Conclusion}

Our finding indicated that H-type HTN was positively associated with the risk of cognitive impairment, especially in $\geq 65$ years old. H-type HTN could be the major intervention measures to decrease the incidence of cognitive impairment as well as the stroke in Health Practice Management.

\section{Introduction}

Cognitive impairment, especially dementia, is common in the elderly and seriously affects the quality of life [1]. Dementia is the most serious expression of cognitive impairment characterized by progressive functional decline in one or more cognitive domains. The prevalence of dementia has increased dramatically, and the number of patients with dementia will reach 130 million in 2050 [2]. The worldwide prevalence of dementia among the elderly was $4.86 \%$ (95\% Cl: $4.20-5.63)$ [3]. In China, there was more than 220 million who aged over 60 was diagnosed with dementia in 2016 [4]. As the aging society intensifies and the number of patients increases, dementia has become a major public health problem. Therefore, dementia is still a progressive, incurable neurodegenerative disorder, and the disability caused by dementia irreversible [5]. 
As for the risk factor of cognitive impairment, previous studies had reported, such as age, cardiovascular disease, genetic factors, diabetes, and so on [6-8]. Interestingly, vascular disorders were reported as modifiable risk factors for cognitive impairment, and hypertension (HTN) plays an important effect in vascular disorders [9]. In order to reduce the risk of cognitive impairment, the World Health Organization (WHO) had set a global target, which was reducing the prevalence of HTN to $25 \%$ in 2025 [10]. An elevated levels of plasma homocysteine( $>15 \propto \mathrm{mol} / \mathrm{L}$ ), known as hyperhomocysteinemia (HHcy), was found to be associated with an increased risk factor for chronic disease, especially among the elderly [11]. Many studies have reported the relationship between hyperuricemia and cognitive impairment, but the conclusions are controversial. Some studies reported that the plasma homocysteine (Hcy) level was associated with the degree of cognitive impairment $[8,12]$. However, some other studies reported that $\mathrm{HTN}$ and HHcy were not associated with the risk of cognitive impairment [13, 14]. H-type hypertension $(\mathrm{H}-$ type HTN), defined as HTN with HHcy, has been received increasing attention recently [15]. As we all known, few studies have examined the association of H-type HTN and cognitive impairment.

Our present cross-sectional study, which was based on a prospective study of Asymptomatic Polyvascular Abnormalities Community Study (APAC), was aimed to explore the relationship between $\mathrm{H}$ type HTN and the risk of cognitive impairment.

\section{Methods}

Study design and population

Participants in the present study were recruited from APAC study. The APAC study is a prospective, observational, community-based long-term cohort study to investigate the epidemiology of asymptomatic polyvascular abnormalities in Chinese adults [16], which was a sub-population of Kailuan study. In 2010, a total of 5440 subjects were enrolled, during the baseline survey, all participants had undergone questionnaire assessment, clinical, and laboratory examinations. The inclusion criteria of APAC study were (1) $\geq 40$ years old ; (2) basic information is complete and available; and (3) free of stroke, myocardial infarction, transient ischemic attack, coronary heart disease and neurological deficits [17]. Among the 5440 participants, we finally identified 3454 observers who had complete information about the mini-mental state examination scores (MMSE), blood pressure and the levels of homocysteine (Fig. 1). Meanwhile, we defined the group older than 65 as elderly, and focused on analyzing the cognitive impairment of the elderly [18].

Assessment of H-type hypertension

H-type HTN was defined as HTN combined with HHcy [19]. The blood pressure of each participant was twice measured by a mercury sphygmomanometer, and the average of the 2 readings was calculated. Hypertension was defined as a systolic blood pressure (SBP) $\geq 140 \mathrm{mmHg}$ or a diastolic blood pressure $(D B P) \geq 90 \mathrm{mmHg}$, a history of hypertension, or taking antihypertensive drugs [20]. 
Plasma total homocysteine (tHcy) concentration was measured by sandwich enzyme-linked immunosorbent assay (Quantikine ELISA, R\&D Systems Inc.). HHcy was defined as the level of plasma homocysteine more than $15 \mu \mathrm{mol} / \mathrm{L}[21,22]$.

Assessment of cognitive impairment

The mini-mental state examination (MMSE) was used as a cognitive screener, involving the ability of time, place, language, construction, learning, calculation, attention, delayed recall and memory [23]. All participants were face-to-face tested the MMSE by specially trained physicians or assistants. The MMSE comprises of 30 items, scores from 0 to 30 points, with a lower score reflecting greater cognitive impairment. MMSE scores was associated with diagnosis of cognitive impairment which was defined as the MMSE scores < 24 [24] .

Definition of different group

Subjects were divided into four groups according to whether they had HTN and HHcy. HTN-/HHcy-: Participants did not have HTN and HHcy; HTN+/HHcy-: Participants have HTN with normal levels of homocysteine; HTN-/HHcy+: Participants have HHcybut with normal blood pressure; $\mathrm{HTN}+/ \mathrm{HHcy+:}$ Participants with H-type HTN (HTN and HHcy).

\section{Covariates}

Demographic information including age, sex, marital status, education level, per capita monthly income, physical activity current smoking status and current alcohol consumption were collected from questionnaires. The body mass index (BMI) was calculated as the weight $(\mathrm{kg})$ divided by the height squared $\left(\mathrm{m}^{2}\right)$. All participants were measured the levels of fasting blood glucose (FBG), total cholesterol (TC), triglycerides (TG), low-density lipoprotein cholesterol (LDL-C) and high-density lipoprotein cholesterol (HDL-C). All blood samples were tested with a Hitachi 747 autoanalyzer (Hitachi; Tokyo, Japan). Diabetes was diagnosed as if $F B G \geq 126 \mathrm{mg} / \mathrm{dl}$ or the participant was receiving treatment for diabetes. Dyslipidemia was defined as TC $\geq 200 \mathrm{mg} / \mathrm{dl}, \mathrm{HDL}-\mathrm{C}<40 \mathrm{mg} / \mathrm{dl}, \mathrm{LDL}-\mathrm{C} \geq 130 \mathrm{mg} / \mathrm{dl}, \mathrm{TG} \geq$ $150 \mathrm{mg} / \mathrm{dl}$, the presence of a history of hyperlipidemia, or drug treatment for dyslipidemia [25].

Statistical analyses

All continuous variables were normal distributed, tested using the Shapiro-Wilk test. Means and standard deviation were calculated for continuous variables. Categorical variables were described with frequencies and percentages and tested with Chi-square tests. Differences among groups were tested by one-way ANOVA for normally distributed variables.

Logistic regression was used to analyze the associations between $\mathrm{HTN}$, HHcy and cognitive impairment with odds ratios (ORs) and 95\% confidence intervals (Cls). The adjustment variables in the model were age, sex, education level, per capita monthly income, current smoking status, alcohol consumption, BMI, diabetes and dyslipidemia. 
Statistical significance was set as $P$ value $<0.05$. All statistical analyses were performed by SAS software, version 9.4 (SAS Institute Inc., Cary, NC, USA).

\section{Results}

\section{Participant and characteristics}

Out of a total of 5440 individuals enrolled in the APAC study, 3454 participants had recorded measurements for blood pressure and the levels of tHcy: $16.79 \%$ of subjects were elderly ( $\geq 65$ years old) and mean age was 53 years (range: $40-89$ years). The prevalence of cognitive impairment, $\mathrm{H}$-type HTN, HTN and HHcy were $4.81 \%, 7.82 \%, 17.28 \%$ and $39.95 \%$, respectively. The characteristic of the participants in our study are summarized in the Table 1 . Significant differences were found in age, gender, education level, physical activity, current smoking, drinking, dyslipidemia, diabetes, BMI and LDL-C during different groups ( all $\mathrm{P} \llbracket 0.05$ ). There were no significant differences in mean or standard deviation or proportion between different groups for marital status, income, TC and HDL-C ( all Pख0.05).

The proportion of cognitive impairment in the H-type HTN (HTN+/HHcy+) group (10.74\%) was higher than other three groups $(2.92 \%, 6.42 \%$ and $5.86 \%$, respectively), and the difference had statistical significance (P凶0.05) (Fig. 2). The prevalence of cognitive impairment in patients with H-type HTN over 65 years old was $22.95 \%$, which was much higher than other characteristic populations ( $\mathrm{P} \otimes 0.001)$.

Associations between HTN, HHcy and cognitive impairment.

Multiple logistic regression analyses showed that participants with HTN had higher risk of cognitive impairment $(\mathrm{OR}=1.84,95 \% \mathrm{Cl}: 1.17-2.90 ; \mathrm{P}<0.001)$, with nonhypertensive participants as reference, after adjusting for multiple variables in model 3 . Similar association was observed in the participants with HHcy $(\mathrm{OR}=1.56,95 \% \mathrm{Cl}$ : 1.02-2.38; $\mathrm{P}<0.001)$ (Fig. 3).

Association between H-type HTN and cognitive impairment risk.

Univariate logistic regression analyses showed that H-type HTN was associated with the risk of cognitive impairment $(\mathrm{OR}=4.00,95 \% \mathrm{Cl}$ : 2.49-6.44; $\mathrm{P} \otimes 0.001)$. After adjusted by confounding factors, H-type HTN remained associated with cognitive impairment $(\mathrm{OR}=2.57,95 \% \mathrm{Cl}: 1.38-4.80 ; \mathrm{P} \otimes 0.001)$. The group of $\mathrm{H}-$ type HTN had the higher risk of cognitive impairment than the isolated HTN group ( $\mathrm{HTN}+$ / HHcy-) (OR = 1.93, 95\% Cl: 1.02-3.70; Pख0.001), when HTN-/HHcy-group as the reference. The isolated HHcy group $(\mathrm{HTN}-/ \mathrm{HHcy}+)$ was no significantly associated with cognitive impairment $(\mathrm{OR}=1.48,95 \% \mathrm{Cl}$ : 0.89-2.46; $\mathrm{P} \otimes 0.05)$ (Fig. 4).

Subgroup analysis of the association of H-type HTN and the risk of cognitive impairment risk.

Age with H-Type HTN was significantly association with cognitive impairment (Fig. 5, P for interaction < 0.001). Stratified analyses showed that the risk of cognitive impairment is higher in elderly H-type hypertensive patients over 65 years old $(\mathrm{OR}=3.63,95 \% \mathrm{Cl}: 1.32-10.01)$. The adjusted $\mathrm{OR}$ and $95 \% \mathrm{Cl}$ for 
cognitive impairment with H-type HTN among participants younger than 65 were 2.01 (0.87-4.67). Similar results were not observed in the different gender of participant. The adjusted ORs and $95 \% \mathrm{Cls}$ for cognitive impairment with H-type HTN in different gender were $2.44(1.19-5.03)$ and 3.14 (0.86-11.42), respectively.

\section{Discussion}

Our study found that H-type HTN was positively associated with the risk of cognitive impairment, in Kailuan community study which was based on the APCP cohort study, especially the participants aged $\geq$ 65 years. In addition, HTN but not isolated HHcy could be an independent risk factors for cognitive impairment.

Previous studies reported that HTN or HHcy were significantly associated with cognitive impairment [26, 27]. To our knowledge, few studies explored the association between $\mathrm{H}$-type HTN and cognitive function. Significant associations have been confirmed between H-type HTN and cardiovascular and cerebrovascular diseases, such as carotid atherosclerotic plaques and stroke [28, 29]. In the present study, we explored the combined association of HTN or HHcy and the risk of cognitive impairment. Our results showed that the ORs of cognitive impairment were increased with the combination of HTN and HHcy develops into H-type HTN. The risk for cognitive impairment in subjects with H-type HTN were 2.6 and 1.4 times with healthy and isolated HTN. Participants were further stratified by gender and age of 65 years (< 65 years old and $\geq 65$ years old). We found that older people over the age of 65 had a higher risk of cognitive impairment. Findings of our study provide the evidence that H-type HTN, the combination of HTN and HHcy, could predict cognitive impairment and may be an independent of other risk factors.

Although there is an agreement that the implication of HTN on the risk of cerebrovascular injury, whether HTN is really harmful to cognitive function remains controversial $[14,26,30]$. When our study was based on a population with non- HTN and non- HHcy and a population without HTN alone, the conclusions were consistent that the damaging effects of HTN on cognition. In addition, HHcy has been reported to contribute to the progression of cognitive impaiment [27]. In our study, the same conclusion was obtained when the non-HHcy population as a reference. However, non-HTN with non-HHcy as reference, we found that isolated HHcy was no significant effect on cognitive impairment.

The predictive role of HTN for cognitive impairment has been widely proven, chronic conditions of HTN lead to deterioration of cognitive impairment in the test that assesses hippocampal functions. HTN could cause damage to the hippocampal and produces typical features such as hypoperfusion, brain amyloid deposition and neuroinflammation. Although the association between HHcy and cognitive impairment is controversial, there have been studies demonstrating the potential mechanisms by which Hcy may induce cerebral arteriosclerosis and catalyze the development of cognitive impairment, such as increased oxidative stress of body tissue, alterations of lipid metabolism, and impaired endothelial function, increase the adhesion of platelets as well as induces the thrombosis [31, 32]. Cerebral arteriosclerosis could induces cerebral infarcts or hemorrhage of varying size and type, attributing to development of 
cognitive impairment [33]. In addition, the synergistic effects of HTN and HHcy may be explained by the fact that HHcy activates the angiotensin-converting enzyme by inhibiting the production of endogenous hydrogen sulfide to lead to or aggravate HTN $[34,35]$. Therefore, when HTN and HHcy are combined, the effects on cognitive impairment may be increased.

In our study, we supplement epidemiological evidence that H-type HTN was an independent risk factor and provides predictive values for cognitive impairment among the elderly. Most of the previous studies analyzed the association between $\mathrm{H}$-type hypertension for cerebrovascular diseases, such as carotid intima-media thickness. To the best of our knowledge, our study was the first community-based and large sample size to assess the potential relationship between $\mathrm{H}$-type hypertension and cognitive impairment.

Despite the large sample size of our study, several limitations should be noted. First, we measured the Hcy concentration but not the Hcy activity in 2012 participant, which might produce a positive bias to evaluate the effect of Hcy. Second, the study was performed based on the participants in China who were 40 years old or older. Therefore, it may be not generalizable for young people who were under the 40 years old. Finally, in this cross-sectional study, we cannot make causal inferences and get more convincing results.

\section{Conclusion}

In summary, our study has provided the evidence for the role of H-type HTN and discussed controversies in relation to HTN or HHcy of cognitive impairment. Our findings indicated that H-type HTN might be a useful marker for cognitive impairment risk assessment especially in elder over 65 . H-type HTN could be the major intervention measures to decrease the incidence of cognitive impairment as well as the stroke in Health Practice Management.

\section{Abbreviations}

APAC, Asymptomatic Polyvascular Abnormalities Community Study. WHO, World Health Organization. HTN, Hypertension; HHcy, hyperhomocysteinemia; H-type HTN, H-type hypertension. MMSE, the minimental state examination. SBP, systolic blood pressure. DBP, diastolic blood pressure. BMI, body mass index; FBG, fasting blood glucose TC, total cholesterol; TG, total cholesterol; HDL-C, high-density lipoprotein cholesterol; LDL-C, low-density lipoprotein cholesterol; HTN+, hypertension; HTN-, without hypertension; $\mathrm{HHcy}+$, homocysteinemia; HHcy-, without homocysteinemia. tHcy, Plasma total homocysteine

\section{Declarations}

\section{Acknowledgements}

We thank all of participants and their relatives who were enrolled in our study. 


\section{Author's contributions}

All authors were involved in the conception or design of the study, data collection, or data analysis. All authors critically reviewed and edited the manuscript and approved the final version for submission.

\section{Funding}

Funding was provided by the National Natural Science Foundation of China (grant/award number: 81773527, 81973138, 91749205 and 81973112), Academic promotion programme of Shandong First Medical University (grant/award number: 2019RC010) and National Key Research and Development Project (grant/award number: 2017YFE0118800)

\section{Availability of data and materials}

The data used to support the findings of this study are available from the corresponding author upon request.

\section{Ethical Approval and Consent to participate}

The study was conducted according to the guidelines of the Helsinki Declaration and was approved by the ethics committee of Kailuan General Hospital of Tangshan City. These approvals will be renewed every five years. Informed consent was obtained from all study subjects.

\section{Consent for publication}

Not applicable

\section{Conflict of interest}

The authors confirmed that there was no conflict of interest.

\section{Authors' information}

${ }^{1}$ School of public health, Shandong First Medical University \& Shandong Academy of Medical Sciences, Taian, Shandong Province, China.

2 Department of Cardiology, Peking University International Hospital. Beijing, China

\section{References}

[1] R. F. de Bruijn, M. J. Bos, M. L. Portegies, A. Hofman, O. H. Franco, P. J. Koudstaal, M. A. Ikram, The potential for prevention of dementia across two decades: the prospective, population-based Rotterdam Study, BMC Med 13 (2015) 132. 
[2] M. Baumgart, H. M. Snyder, M. C. Carrillo, S. Fazio, H. Kim, H. Johns, Summary of the evidence on modifiable risk factors for cognitive decline and dementia: A population-based perspective, Alzheimers Dement 11(6) (2015) 718-26.

[3] Y. Zhu, H. Liu, X. L. Lu, B. Zhang, W. Weng, J. Yang, J. Zhang, M. J. Dong, Prevalence of dementia in the People's Republic of China from 1985 to 2015: a systematic review and meta-regression analysis, BMC Public Health 19(1) (2019) 578.

[4] X. Xu, L. Chen, Influencing factors of disability among the elderly in China, 2003-2016: application of Bayesian quantile regression, J Med Econ 22(6) (2019) 605-611.

[5] T. P. Lam, K. S. Sun, H. Y. Chan, C. S. Lau, K. F. Lam, R. Sanson-Fisher, Perceptions of Chinese Towards Dementia in Hong Kong-Diagnosis, Symptoms and Impacts, Int J Environ Res Public Health 16(1) (2019).

[6] M. M. Santisteban, C. ladecola, Hypertension, dietary salt and cognitive impairment, J Cereb Blood Flow Metab 38(12) (2018) 2112-2128.

[7] L. Vermunt, S. A. M. Sikkes, A. van den Hout, R. Handels, I. Bos, W. M. van der Flier, S. Kern, P. J. Ousset, P. Maruff, I. Skoog, F. R. J. Verhey, Y. Freund-Levi, M. Tsolaki, A. K. Wallin, M. Olde Rikkert, H. Soininen, L. Spiru, H. Zetterberg, K. Blennow, P. Scheltens, G. Muniz-Terrera, P. J. Visser, Initiative Alzheimer Disease Neuroimaging, Aibl Research Group, Ictus Dsa study groups, Duration of preclinical, prodromal, and dementia stages of Alzheimer's disease in relation to age, sex, and APOE genotype, Alzheimers Dement 15(7) (2019) 888-898.

[8] S. Tian, J. Han, R. Huang, J. Sun, R. Cai, Y. Shen, S. Wang, Increased Plasma Homocysteine Level is Associated with Executive Dysfunction in Type 2 Diabetic Patients with Mild Cognitive Impairment, J Alzheimers Dis 58(4) (2017) 1163-1173.

[9] P. B. Gorelick, A. Scuteri, S. E. Black, C. Decarli, S. M. Greenberg, C. ladecola, L. J. Launer, S. Laurent, O. L. Lopez, D. Nyenhuis, R. C. Petersen, J. A. Schneider, C. Tzourio, D. K. Arnett, D. A. Bennett, H. C. Chui, R. T. Higashida, R. Lindquist, P. M. Nilsson, G. C. Roman, F. W. Sellke, S. Seshadri, Council on Epidemiology American Heart Association Stroke Council, Council on Cardiovascular Nursing Council on Cardiovascular Radiology Prevention, Intervention, Surgery Council on Cardiovascular, Anesthesia, Vascular contributions to cognitive impairment and dementia: a statement for healthcare professionals from the american heart association/american stroke association, Stroke 42(9) (2011) 2672-713.

[10] S. Cahill, WHO's global action plan on the public health response to dementia: some challenges and opportunities, Aging Ment Health (2019) 1-3.

[11] P. K. Kamat, P. Kyles, A. Kalani, N. Tyagi, Hydrogen Sulfide Ameliorates Homocysteine-Induced Alzheimer's Disease-Like Pathology, Blood-Brain Barrier Disruption, and Synaptic Disorder, Mol Neurobiol 53(4) (2016) 2451-2467. 
[12] S. Kim, B. Y. Choi, J. H. Nam, M. K. Kim, D. H. Oh, Y. J. Yang, Cognitive impairment is associated with elevated serum homocysteine levels among older adults, Eur J Nutr 58(1) (2019) 399-408.

[13] K. Nilsson, L. Gustafson, B. Hultberg, Elevated plasma homocysteine level is not primarily related to Alzheimer's disease, Dement Geriatr Cogn Disord 34(2) (2012) 121-7.

[14] M. Lazo-Porras, V. Ortiz-Soriano, M. Moscoso-Porras, F. M. Runzer-Colmenares, G. Malaga, J. Jaime Miranda, Cognitive impairment and hypertension in older adults living in extreme poverty: a crosssectional study in Peru, BMC Geriatr 17(1) (2017) 250.

[15] H. Wang, Z. Li, X. Guo, Y. Chen, S. Chen, Y. Tian, Y. Sun, Contribution of non-traditional lipid profiles to reduced glomerular filtration rate in H-type hypertension population of rural China, Ann Med 50(3) (2018) 249-259.

[16] H. Liu, Y. Yao, Y. Wang, L. Ji, K. Zhu, H. Hu, J. Chen, J. Yang, Q. Cui, B. Geng, Q. Liu, D. Li, Y. Zhou, Association between high-sensitivity C-reactive protein, lipoprotein-associated phospholipase A2 and carotid atherosclerosis: A cross-sectional study, J Cell Mol Med 22(10) (2018) 5145-5150.

[17] A. Wang, Z. Su, X. Liu, Y. Yang, S. Chen, S. Wang, Y. Luo, X. Guo, X. Zhao, S. Wu, Brachial-ankle pulse wave velocity and metabolic syndrome in general population: the APAC study, BMC Cardiovasc Disord 16(1) (2016) 228.

[18] J. R. Beard, A. M. Officer, A. K. Cassels, The World Report on Ageing and Health, Gerontologist 56 Suppl 2 (2016) S163-6.

[19] J. Zhang, Y. Liu, A. Wang, D. Wang, R. Jiang, J. Jia, S. Chen, X. Zhao, Association between H-type Hypertension and Asymptomatic Extracranial Artery Stenosis, Sci Rep 8(1) (2018) 1328.

[20] J. Wang, Y. Liu, L. Zhang, N. Li, C. Wang, X. Gao, Y. Zhou, A. Wang, S. Wu, X. Zhao, Associations of high sensitivity C-reactive protein levels with the prevalence of asymptomatic intracranial arterial stenosis, Eur J Neurol 21(3) (2014) 512-8.

[21] J. M. Sabio, J. A. Vargas-Hitos, J. Martinez-Bordonado, N. Navarrete-Navarrete, A. Diaz-Chamorro, C. Olvera-Porcel, M. Zamora-Pasadas, J. Jimenez-Alonso, Relationship between homocysteine levels and hypertension in systemic lupus erythematosus, Arthritis Care Res (Hoboken) 66(10) (2014) 1528-35.

[22] P. A. Zena-Huancas, H. Iparraguirre-Lopez, R. V. Gamboa-Cardenas, C. Reategui-Sokolova, F. ZevallosMiranda, M. Medina-Chinchon, V. R. Pimentel-Quiroz, C. Elera-Fitzcarrald, O. Sarmiento-Velasquez, J. M. Cucho-Venegas, J. L. Alfaro-Lozano, Z. J. Rodriguez-Bellido, C. A. Pastor-Asurza, R. A. Perich-Campos, G. S. Alarcon, M. F. Ugarte-Gil, Homocysteine levels are independently associated with damage accrual in systemic lupus erythematosus patients from a Latin-American cohort, Clin Rheumatol 38(4) (2019) 11391146. 
[23] A. Wang, R. Jiang, Z. Su, J. Jia, N. Zhang, J. Wu, S. Chen, X. Zhao, A low ankle-brachial index is associated with cognitive impairment: The APAC study, Atherosclerosis 255 (2016) 90-95.

[24] N. I. Wellens, J. Flamaing, J. Tournoy, T. Hanon, P. Moons, G. Verbeke, S. Boonen, K. Milisen, Convergent validity of the Cognitive Performance Scale of the interRAl acute care and the mini-mental state examination, The American journal of geriatric psychiatry : official journal of the American Association for Geriatric Psychiatry 21(7) (2013) 636-45.

[25] N. Zhang, Y. Yang, A. Wang, Y. Cao, J. Li, Y. Yang, K. Zhang, W. Zhang, S. Wu, Z. Wang, M. Zhu, Y. Zhang, S. Wu, C. Wang, X. Zhao, Association of ideal cardiovascular health metrics and cognitive functioning: the APAC study, Eur J Neurol 23(9) (2016) 1447-54.

[26] C. ladecola, R. F. Gottesman, Neurovascular and Cognitive Dysfunction in Hypertension, Circ Res 124(7) (2019) 1025-1044.

[27] F. Zhou, S. Chen, Hyperhomocysteinemia and risk of incident cognitive outcomes: An updated doseresponse meta-analysis of prospective cohort studies, Ageing Res Rev 51 (2019) 55-66.

[28] Z. Chen, F. Wang, Y. Zheng, Q. Zeng, H. Liu, H-type hypertension is an important risk factor of carotid atherosclerotic plaques, Clin Exp Hypertens 38(5) (2016) 424-8.

[29] H. Pang, B. Han, Q. Fu, Z. Zong, Association of High Homocysteine Levels With the Risk Stratification in Hypertensive Patients at Risk of Stroke, Clin Ther 38(5) (2016) 1184-92.

[30] J. Park, J. W. Kim, B. Ryu, E. Heo, S. Y. Jung, S. Yoo, Patient-Level Prediction of CardioCerebrovascular Events in Hypertension Using Nationwide Claims Data, J Med Internet Res 21(2) (2019) e11757.

[31] J. David Spence, Homocysteine-lowering therapy: a role in stroke prevention?, The Lancet Neurology 6(9) (2007) 830-838.

[32] R. Moretti, P. Caruso, The Controversial Role of Homocysteine in Neurology: From Labs to Clinical Practice, Int J Mol Sci 20(1) (2019).

[33] Saito S, Yamamoto Y, Ihara M \%J Current Alzheimer research, Mild Cognitive Impairment: At the Crossroad of Neurodegeneration and Vascular Dysfunction, 12(6) (2015) 507-12.

[34] C. Antoniades, A. S. Antonopoulos, D. Tousoulis, K. Marinou, C. Stefanadis, Homocysteine and coronary atherosclerosis: from folate fortification to the recent clinical trials, Eur Heart J 30(1) (2009) 615.

[35] H. Laggner, M. Hermann, H. Esterbauer, M. K. Muellner, M. Exner, B. M. Gmeiner, S. Kapiotis, The novel gaseous vasorelaxant hydrogen sulfide inhibits angiotensin-converting enzyme activity of endothelial cells, Journal of hypertension 25(10) (2007) 2100-4. 


\section{Table}

Due to technical limitations, table 1 is only available as a download in the supplemental files section. Figures

\section{Figure 1}

Flowchart of the study. *APAC, Asymptomatic Polyvascular Abnormalities Community; MMSE, minimental state examination; $\mathrm{HTN}+$, hypertension; $\mathrm{HTN}-$, without hypertension; $\mathrm{HHcy}+$, homocysteinemia; HHcy-, without homocysteinemia.

\section{Figure 2}

The proportion of participants with cognitive impairment between genders in the study groups. *HTN+, hypertension; $\mathrm{HTN}-$-, without hypertension; HHcy+, homocysteinemia; $\mathrm{HHcy}-$, without homocysteinemia.

\section{Figure 3}

Association of hypertension with hyperhomocysteinemia and cognitive impairment. *Model 1: unadjusted model; Model 2: adjusted for age, sex, education, current smoking, alcohol consumption, physical activity. Model 3: adjusted for model 2 plus hyperlipidemia, diabetes mellitus, and BMI.

\section{Figure 4}

Association of $\mathrm{H}$-type hypertension and cognitive impairment. *HTN+, hypertension; $\mathrm{HTN}$-, without hypertension; HHcy+, homocysteinemia; HHcy-, without homocysteinemia; Hypertension was defined as systolic pressure $>140 \mathrm{mmHg}$ or diastolic pressure $>90 \mathrm{mmHg}$. Homocysteinemia was defined as the level of plasma homocysteine $>15 \mu \mathrm{mol} / \mathrm{L}$.

\section{Figure 5}

Association of $\mathrm{H}$-type hypertension and cognitive impairment between different ages and genders in our study. *adjusted for age, sex, education, current smoking, alcohol consumption, physical activity, hyperlipidemia, diabetes mellitus, and BMI. ${ }^{\star *} \mathrm{P}$ for interaction $<0.001$.

\section{Supplementary Files}


This is a list of supplementary files associated with this preprint. Click to download.

- table1.pdf 